\title{
Laminar Forced Convection Modeling of Isothermal Rectangular Plates
}

\author{
M. M. Yovanovich* and P. Teertstra ${ }^{\dagger}$ \\ University of Waterloo, Waterloo, Ontario N2L 3G1, Canada
}

\begin{abstract}
A composite model for area-average Nusselt number for forced, laminar flow parallel to a finite, isothermal rectangular plate for a wide range of Reynolds numbers is proposed. The correlation equation is based on the superposition of the dimensionless shape factor and a modified laminar flow boundary-layer asymptote with an empirically determined interpolation parameter. The Nusselt and Reynolds numbers and the dimensionless shape factor are based on either the rectangle side dimension parallel to the flow direction or the square root of the heat transfer area. The proposed correlation equations are applicable to rectangles with side dimension ratios in a range from 1 to 10. Extensive numerical results were used to find the optimal values of the interpolation parameter to provide close agreement between the correlation equation predictions and the numerical values.
\end{abstract}

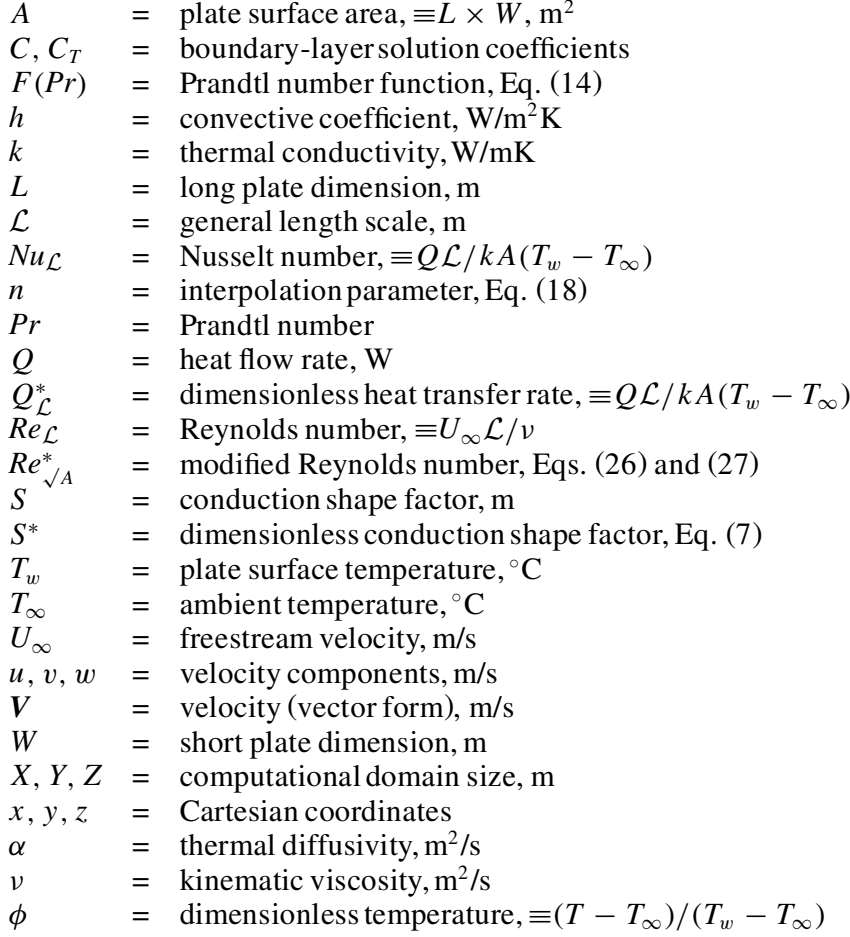

Subscripts

$\begin{array}{ll}\sqrt{ } A & =\text { with square root of plate area } A \\ L & =\text { with long plate dimension } \\ W & =\text { with short plate dimension } \\ w & =\text { plate surface } \\ \infty & =\text { freestream }\end{array}$

\section{Introduction}

$\mathbf{L}$ AMINAR forced convective heat transfer from isothermal finite rectangular plates over a range of Reynolds number such

Received 28 January 2000; revision received 9 October 2000; accepted for publication 13 November 2000. Copyright (C) 2001 by M. M. Yovanovich and P. Teertstra. Published by the American Institute of Aeronautics and Astronautics, Inc., with permission.

* Distinguished Professor Emeritus, Microelectronics Heat Transfer Laboratory, Department of Mechanical Engineering. Fellow AIAA.

${ }^{\dagger}$ Graduate Research Assistant, Microelectronics Heat Transfer Laboratory, Department of Mechanical Engineering. as $1 \leq R e \leq 5000$ is a problem that has not been addressed fully in the open literature. Correlations for the local and average Nusselt number are limited to two-dimensional laminar boundary-layer flow, such as the Blasius-Pohlhausen solution (see Refs. 1-3) and are available for a limited Reynolds number range, such as $100<R e<10^{5}$. Therefore, they cannot be used for finite plates at small Reynolds numbers, where thermal diffusion becomes important and the resulting temperature field is three dimensional.

A composite model for the Nusselt number that is reported to be valid for a wide range of Reynolds and Prandtl numbers was published recently. ${ }^{4}$ The correlation equation includes the diffusive limit for zero fluid flow and the boundary-layerlimit that accounts for turbulent effects. The composite model is based on the linear superposition of the two limits, where the square root of the total surface area is used as the length scale. However, this model is limited to two-dimensional boundary-layer flow and is not applicable to the finite rectangular plate problem.

The major objectives of this paper are 1) to develop a composite correlation equation that accurately predicts the average Nusselt number for all fluids $\operatorname{Pr}>0.5$ for a range of the Reynolds number $\left.0<R e_{\mathcal{L}}<5000,2\right)$ to examine the effect of the length scale used in the dimensionless parameters such as the Nusselt and Reynolds numbers and the shape factor, and 3) to report accurate numerical values of the Nusselt number for the finite rectangular plate over a wide range of Reynolds numbers and aspect ratios.

\section{Physical Problem Description}

Consider steady, laminar flow of a constant property fluid of large extent and temperature $T_{\infty}$ parallel to a finite isothermal rectangular plate of zero thickness and side dimensions $L$ and $W$, where $L / W \geq 1$. The upper surface is maintained at temperature $T_{w}$ and the lower surface of the plate is assumed to be adiabatic, such that the total heat transfer area is $A=L W$. Two flow cases are considered as shown in Fig. 1: Fig. 1a shows flow parallel to the longer side dimension $L$ and Fig. $1 \mathrm{~b}$ flow parallel to the shorter side dimension $W$. The ratio of the side dimensions of the rectangular plate ranges between 1 for the square plate and 10 for a long rectangular plate. The Reynolds number based on the freestream velocity $U_{\infty}$ and the plate dimension parallel to the flow direction will range from 0.1 up to 5000. As the Reynolds number increases from small to large values, the local heat flux distribution changes between two cases. When the Reynolds number is very small, the heat flux attains its maximum values along the four edges of the rectangular plate. For larger values of the Reynolds number the maximum values of the local heat flux occur along the leading edge, where the fluid first encounters the plate. 


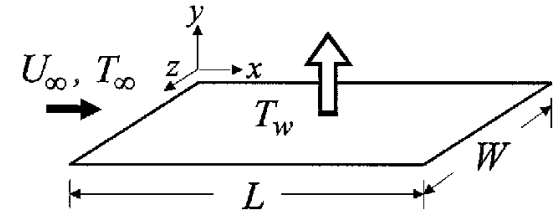

a) Flow along long plate dimension $L$

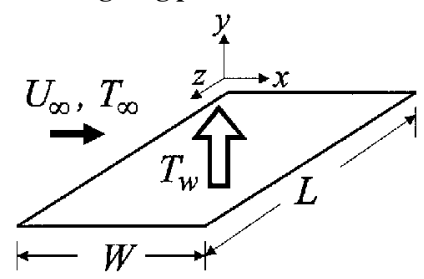

b) Flow along short plate dimension $W$

Fig. 1 Schematics of physical problem.

\section{Mathematical Problems}

The governing differential equations in vector form for the full three-dimensional problem are the continuity equation,

$$
\nabla \cdot \boldsymbol{V}=0
$$

the momentum equation for zero pressure gradient along the plate surface,

$$
v \nabla^{2} \boldsymbol{V}=(\boldsymbol{V} \cdot \nabla) \boldsymbol{V}
$$

and the energy equation with negligible viscous heating,

$$
\alpha \nabla^{2} T=(\boldsymbol{V} \cdot \nabla) T
$$

where the Laplacian operator in Cartesian coordinates is

$$
\nabla^{2}=\frac{\partial^{2}}{\partial x^{2}}+\frac{\partial^{2}}{\partial y^{2}}+\frac{\partial^{2}}{\partial z^{2}}
$$

The constant thermophysical properties that appear in the momentum and energy equations are the kinematic viscosity $v$ and the thermal diffusivity $\alpha$, respectively. It has been assumed that there is negligible viscous heating because the flow velocity is small. All buoyancy effects have been neglected, such that as $R e_{\mathcal{L}} \rightarrow 0$ the velocity at all points in the fluid approaches 0 and heat transfer occurs by conduction alone.

There are no analytical solutions of the Navier-Stokes equations available for the full range of the Reynolds number from small values, $R e_{\mathcal{L}}=0$, to large values, $R e_{\mathcal{L}}=5000$. There are, however, two well-known limiting cases. When the Reynolds number lies in the range $100<\operatorname{Re}_{\mathcal{L}}<10^{5}$ and the thermal and hydrodynamic boundary-layer thicknesses are much smaller than the plate dimensions $L$ and $W$, the Navier-Stokes equations reduce to the two-dimensional boundary-layerequations. The solution of the boundary-layer form of the equations for the flat plate has been well documented in the literature. ${ }^{1-3}$ When the Reynolds number approaches very small values such as $R e_{\mathcal{L}}<0.1$, the solution of the energy equation will approach the solution of the three-dimensional Laplace equation

$$
\nabla^{2} T=0
$$

For all problems, the dimensionless heat transfer rate from the surface of the isothermal rectangularplate is obtained from the relation

$$
Q_{\mathcal{L}}^{*}=\frac{Q \mathcal{L}}{k A\left(T_{w}-T_{\infty}\right)}=\frac{\mathcal{L}}{A} \int_{0}^{W} \int_{0}^{L}-\frac{\partial \phi}{\partial y} \mathrm{~d} x \mathrm{~d} z
$$

where $Q$ is the total heat transfer rate from the plate, $\mathcal{L}$ is some characteristic length scale, $A$ is the total surface area, and $T_{w}$ and $T_{\infty}$ are the temperatures of the plate and the fluid at points remote from the center of the plate. The dimensionless temperature is $\phi=\left[T(x, y, z)-T_{\infty}\right] /\left(T_{w}-T_{\infty}\right)$. The thermal and hydrodynamic boundary conditions on the surface of the plate are $T=T_{w}$ and $u=v=w=0$. At points remote from the center of the plate, $T \rightarrow T_{\infty}$ and $u \rightarrow U_{\infty}$, and both velocity components $v$ and $w$ go to 0 .

\section{Solutions of the Limiting Problems}

Analytical solutionsto the full equations for arbitrary values of the Reynolds number are not available. Results of the available analytical and numerical solutions for limiting cases of the full equations are considered in the following sections. These relations will be used in subsequent sections to develop a composite model for the dimensionless heat transfer rate over a wide range of the Reynolds number.

\section{Diffusive Limit Solution}

The dimensionless heat transfer rate for zero velocity (called the diffusive limit) is obtained from the solution of the threedimensional Laplace equation. The dimensionless heat transfer rate for this limit is called the dimensionless shape factor, ${ }^{5}$ defined as

$$
Q_{\mathcal{L}}^{*}=S_{\mathcal{L}}^{*}=S \mathcal{L} / A
$$

for the limit $R e_{\mathcal{L}} \rightarrow 0$. Analytical solutions for the conduction shape factor for the isothermal rectangular plate are not available; however, Yovanovich ${ }^{5}$ has shown that the analytical solution for the isothermal elliptical disk can be used to approximate the numerical results ${ }^{6}$ for the isothermal rectangular plate with acceptable accuracy. This approximate solution requires that the heat transfer areas and the aspect ratios of the rectangular plate and the elliptical disk are similar and that the characteristic length scale is based on the square root of the total active area. The recommended relations for the dimensionless shape factor for the isothermal rectangular plate $\operatorname{are}^{5}$

$$
S_{\sqrt{A}}^{*}=\frac{(1+\sqrt{L / W})^{2}}{\sqrt{\pi L / W}}, \quad 1 \leq L / W \leq 5
$$

and

$$
S_{\sqrt{A}}^{*}=\frac{2 \sqrt{\pi L / W}}{\ln (4 L / W)}, \quad 5<L / W<\infty
$$

The dimensionless shape factor depends weakly on the rectangle aspect ratio $L / W \geq 1$ when $\mathcal{L}=\sqrt{ } A$. The maximum difference between the predicted values and previously determined numerical values $^{6}$ of $S^{*}{ }$ A for the range $1 \leq L / W \leq 4$ is less than $1 \%$. It is expected that the two relations should provide values that are within $\pm 3 \%$ of the numerical estimates for values of $L / W>4$. When the side length dimensions $L$ or $W$ are selected as the length scale, the dimensionless shape factors are obtained from

$$
\begin{aligned}
& S_{L}^{*}=\sqrt{L / W} S_{\sqrt{A}}^{*} \\
& S_{W}^{*}=\sqrt{W / L} S_{\sqrt{A}}^{*}
\end{aligned}
$$

\section{Boundary-Layer Solutions}

The two-dimensional boundary layer equations were solved by Blasius and Pohlhausen (see Ref. 1), and these solutions are presented in many fluids and heat transfer texts. ${ }^{1-3}$ For laminar flow over an isothermal plate of length $L$, the area-average Nusselt number for $\operatorname{Pr}>0.5$ and $100<R e_{L}<10^{5}$ is given by

$$
N u_{L}=C \operatorname{Re}_{L}^{\frac{1}{2}} \operatorname{Pr}^{\frac{1}{3}}
$$

where the most frequently quoted value for the coefficient is $C=0.664$. According to Kays and Crawford, ${ }^{3}$ the true value of the coefficient for the $\operatorname{Pr} \rightarrow \infty$ limit is $C=0.677$. Yovanovich et al. ${ }^{7}$ obtained an approximate analytical solution that is based 
on a linearization of the momentum and energy equations. They reported that the average Nusselt number can be obtained from

$$
N u_{L}=2 F(\operatorname{Pr}) \operatorname{Re}_{L}^{\frac{1}{2}}
$$

where the Prandtl number function is defined as

$$
F(P r)=\left\{\operatorname{Pr} / \pi\left[1+\left(C_{T} \operatorname{Pr}^{\frac{1}{3}}\right)^{2}\right]^{\frac{1}{2}}\right\}^{\frac{1}{2}}
$$

which is valid for $0<\operatorname{Pr}<\infty$. The limiting values of the Prandtl number function were reported as

$$
\begin{array}{ll}
F(\operatorname{Pr}) \rightarrow(1 / \sqrt{\pi}) \sqrt{P r}, & \operatorname{Pr} \rightarrow 0 \\
F(\operatorname{Pr}) \rightarrow\left(1 / \sqrt{\pi C_{T}}\right) \operatorname{Pr}^{\frac{1}{3}}, & \operatorname{Pr} \rightarrow \infty
\end{array}
$$

The numericalvalue of the constant $C_{T}$ that appears in the linearized energy equation can be determined in two ways. When the wall heat flux of the proposed linearized model is matched against the value obtained from Pohlhausen (see Ref. 1), a value of $C_{T}=2.77$ is obtained. Matching the enthalpy flux into the thermal boundary layer predicted by the proposed model against the value obtained by means of the integral energy equation solution results in a lower value of the coefficient, $C_{T}=2.13$. When a value of $C_{T}=2.31$ is assumed, which is close to various averages of these two values, the linearized model gives a boundary-layer solution identical in form to Pohlhausen (see Ref. 1) except for a larger coefficient, $C=0.742$.

\section{Proposed Composite Model}

The proposed model consists of two components, the laminar boundary-layersolution and the solution for thermal diffusion. The laminar boundary-layersolution has the form

$$
N u_{\mathcal{L}}=0.742 \operatorname{Re}_{\mathcal{L}}^{\frac{1}{2}} \operatorname{Pr}^{\frac{1}{3}}
$$

which is valid for $\operatorname{Pr}>0.5$ and $100<\operatorname{Re}_{\mathcal{L}}<10^{5}$. In the proposed boundary-layer solution, the Nusselt and Reynolds numbers are based on the arbitrary length scale $\mathcal{L}$. The conventional characteristic length used in the Nusselt and Reynolds numbers is the length of the plate in the flow direction, $L$ or $W$. The appropriate length scale will arise from the subsequent analysis.

The proposed composite correlation equation is based on the Churchill-Usagi ${ }^{8}$ method of combining asymptotic solutions

$$
N u_{\mathcal{L}}=\left[\left(S_{\mathcal{L}}^{*}\right)^{n}+\left(0.742 \operatorname{Re}_{\mathcal{L}}^{\frac{1}{2}} \operatorname{Pr}^{\frac{1}{3}}\right)^{n}\right]^{1 / n}
$$

where the interpolation parameter $n$ ensures that the model predicts accurate values of the Nusselt number for intermediate values of the Reynolds number. The interpolation parameter is expected to be dependent on the aspect ratio of the rectangular plate when the conventionallength scales are used in the Nusselt and Reynolds numbers and the dimensionless shape factor. The appropriate values of this parameter will be found by fitting the proposed model to numerical values obtained by means of a well-established commercial computational fluid dynamics (CFD) code.

\section{Numerical Procedure}

The analytical forced convection model developed in the preceding section was validated and optimized using results obtained from simulations performed using FLOTHERM, ${ }^{9}$ a commercial finite volume CFD software package. The FLOTHERM package utilizes the standard finite volume analysis techniques as presented by Patankar. ${ }^{10}$ These CFD simulations were used to model the fluid flow and heat transfer within an air-filled region surrounding the isothermal plate, as shown in Figs. 2a and 2b. The physical models selected for the numerical solutions were steady-state, laminar flow, with constantfluid properties, no buoyancy forces, and no radiation.

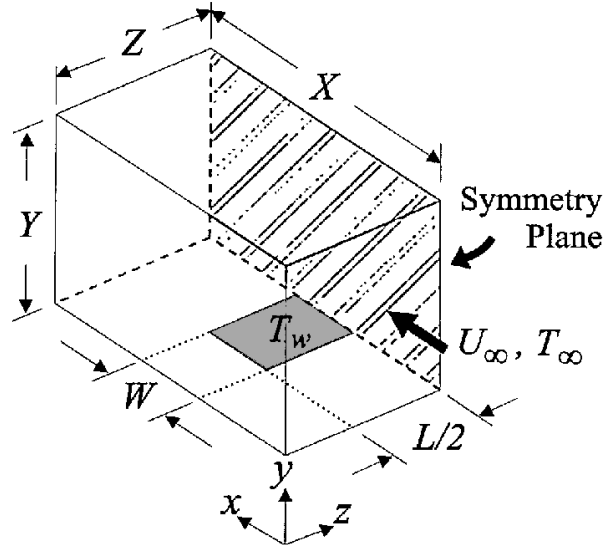

a) Flow along short plate dimension $W$

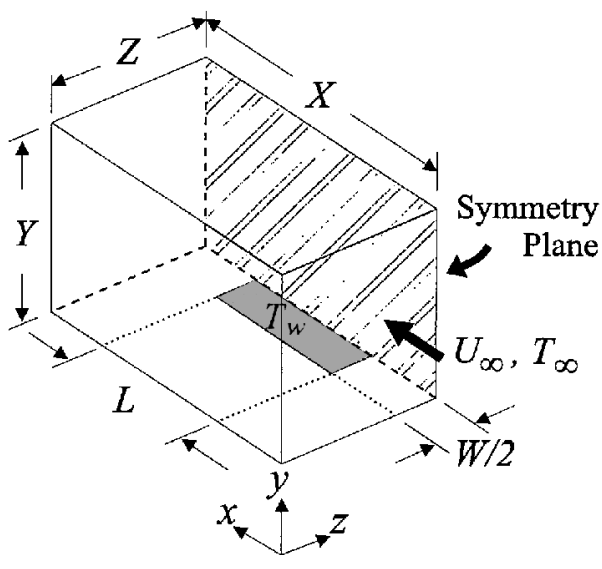

b) Flow along long plate dimension $L$

Fig. 2 Schematics of CFD solution domain.

The flat plate was modeled as an isothermal, no-slip boundary in contact with the moving fluid. An adiabatic, zero-shear symmetry boundary condition was applied along the midplane of the plate in the flow direction as shown in Fig. 2. The uniform freestream velocity $U_{\infty}$ and ambient temperature $T_{\infty}$ were specified at the upstream boundary, and the downstream and lateral domain boundaries were set to atmospheric (zero) pressure, allowing heat and mass to exit freely from the system. The plate and ambient air temperatures were set to $T_{w}=40^{\circ} \mathrm{C}$ and $T_{\infty}=20^{\circ} \mathrm{C}$, respectively, and constant air properties evaluated at the film temperature $300 \mathrm{~K}$ were assumed.

Because of the large range of Reynolds number proposedfor these simulations, $1 \leq R e \leq 5000$, it was anticipated that different computational domain sizes would be required, depending on the Reynolds number. For small Reynolds number, $R e<10$, the heat transfer is dominated by conduction, requiring a large computational domain to model a fluid region of infinite extent. This same solution domain is also valid for the diffusive limit, the limiting case when $R e \rightarrow 0$, where heat transfer is by conduction only. At the large Reynolds number limit, $R e>1000$, the majority of the heat transfer from the plate is by convection through a thin laminar boundary layer. At this limit, the size of the domain can be substantially reduced, but many more control volumes concentrated near the plate surface are necessary to resolve accurately the large temperature gradients. The dimensions of the solution domain in the $x, y$, and $z$ directions are characterized by $X, Y$, and $Z$, respectively, as shown in Fig. 2. Typical values for these dimensions used in the CFD simulations, nondimensionalizedusing the plate length $L$, are presented in Table 1.

To generate accurate results using the CFD model, it is necessary to demonstrate that the effects of the size and number of control volumes on the solution have been minimized. Because of the distinct differences between the computational domains for small and large Reynolds numbers, two grid convergence studies were performed. 
Table 1 Typical CFD solution domain dimensions

\begin{tabular}{lccc}
\hline \hline$R e$ & $X / L$ & $Y / L$ & $Z / L$ \\
\hline$\rightarrow 0$ & 100 & 50 & 50 \\
1 & 100 & 50 & 50 \\
10 & 100 & 50 & 50 \\
100 & 50 & 20 & 20 \\
1000 & 15 & 1.5 & 7 \\
\hline \hline
\end{tabular}

Table 2 Summary of test cases for CFD simulations

\begin{tabular}{|c|c|c|}
\hline$L / W$ & $\begin{array}{c}\text { Flow } \\
\text { direction }\end{array}$ & $R e$ \\
\hline 10 & $W$ & $\rightarrow 0,1,10,100,500$ \\
\hline 5 & $W$ & $\begin{array}{l}\rightarrow 0,1,2,4,10,20,40,100 \\
\quad 200,400,1000\end{array}$ \\
\hline 2 & $W$ & $\begin{array}{l}\rightarrow 0,1,2.5,5,10,25,50 \\
\quad 100,250,500,1000,2500\end{array}$ \\
\hline 1 & $L$ & $\begin{array}{l}\rightarrow 0,1,2,5,10,20,50,100 \\
\quad 200,500,1000,2717.3,5000\end{array}$ \\
\hline 2 & $L$ & $\begin{array}{c}\rightarrow 0,1,2,5,10,20,50,100 \\
\quad 200,500,1000,2000,5000\end{array}$ \\
\hline 5 & $L$ & $\begin{array}{r}\rightarrow 0,1,2,5,10,20,50,100 \\
200,500,1000,2000,5000\end{array}$ \\
\hline 10 & $L$ & $\rightarrow 0,1,10,100,1000$ \\
\hline
\end{tabular}

For the square plate with $R e=2717.3$, two test cases were examined. The refined grid used in the second case had a 5.3 times increase in the number of control volumes over the first, to 144,000 , and a $60 \%$ reduction in the thickness of the first layer of control volumes in contact with the plate surface. The resulting change in Nusselt number was only $0.5 \%$, indicating that a converged solution had been achieved. The finer grid from the second test case was used for all remaining large Reynolds number simulations.

The second grid convergence study involved two test cases for the diffusive limit for $L / W=5$ with flow parallel to the long side length $L$. Refining the grid by a 3.3 times increase in the number of control volumes and a $40 \%$ reduction in the thickness of the first layer of control volumes in contact with the plate surface resulted in a change in Nusselt number of $2.4 \%$. This small change in Nusselt number for a relatively large change in grid parameters indicated that convergence had been achieved, and the refined grid was used in all remaining small Reynolds number simulations.

The accuracy of the numerical results can be verified by comparison with the available exact solutions at the two limiting cases, the diffusive limit and the laminar boundary-layer limit. For the diffusive limit, the result from the refined grid for $L / W=5$ was within $0.6 \%$ of the value predicted by the available analytical expression [Eq. (10)]. For the large Reynolds number, $R e=5000$, the numerical prediction for $L / W=1$ was within $0.7 \%$ of the value resulting from the laminar boundary-layersolution. This excellent agreement between the CFD results and the exact solutions at the two limiting cases validated the accuracy of the numerical predictions.

\section{Numerical Results and Discussion}

With the size and discretization of the computation domain established, the CFD model was used to simulate a wide range of aspect ratios and Reynolds numbers, for both flow along the long plate dimension $L$ and the short plate dimension $W$, as shown in Table 2 .

The numerical results for these test cases are compared with the predictions of the proposed model [Eq. (18)] in Fig. 3. The data are nondimensionalizedusing Nusselt and Reynolds numbers defined as follows. For the bulk velocity parallel to the short platedimension $W$,

$$
\begin{gathered}
N u=N u_{W}=\frac{Q W}{k A\left(T_{w}-T_{\infty}\right)} \\
R e=R e_{W}=\frac{U_{\infty} W}{v}
\end{gathered}
$$

and for flow parallel to the long plate dimension $L$,

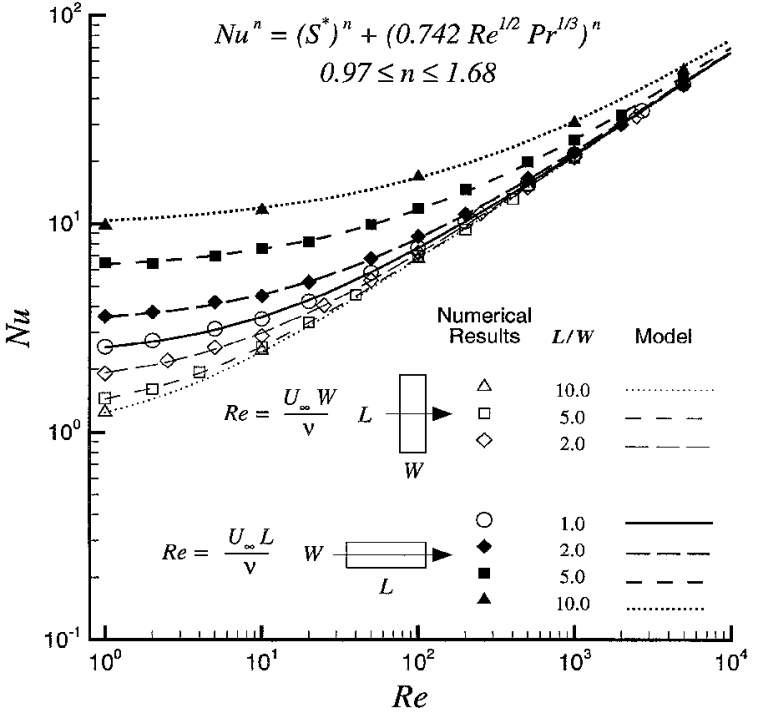

Fig. 3 Comparison of data and model with $L$ and $W$ as characteristic length.

$$
\begin{gathered}
N u=N u_{L}=\frac{Q L}{k A\left(T_{w}-T_{\infty}\right)} \\
R e=R e_{L}=U_{\infty} L / v
\end{gathered}
$$

The data are compared with the following form of the proposed model:

$$
N u=\left[\left(S^{*}\right)^{n}+\left(0.742 \operatorname{Re}^{\frac{1}{2}} \operatorname{Pr}^{\frac{1}{3}}\right)^{n}\right]^{1 / n}
$$

where Nusselt number and Reynolds number are defined in Eqs. (19-22). The conduction shape factor $S^{*}$ is determined by Eq. (11) for flow along the short plate dimension $W$ and by Eq. (10) for flow parallel to $L$.

When the numerical data were used, optimized values for the interpolation parameter $n$ were determined that minimized the deviation between the proposed model and the data. The resulting interpolation parameters for all test cases vary according to aspect ratio and flow direction in the range

$$
0.97 \leq n \leq 1.68
$$

These optimized values for the interpolation parameter $n$ were correlated separately for each flow direction as a function of the aspect ratio. For bulk velocity parallel to the short plate dimension $W$,

$$
n=1.42-0.28 \log _{10}(W / L)
$$

and for flow along the long plate dimension $L$,

$$
n=1.42-0.45 \log _{10}(L / W)
$$

For the square plate where $L=W$, both correlation equations provide the same value. When the proposed model [Eq. (23)] and the interpolation parameter $n$ from the appropriate correlation equation (24) or (25) are used, the numerical results are predicted within a maximum percent difference of $4.5 \%$ and an rms percent difference of $1.9 \%$.

The large variation in the values of the interpolationparameter for the different aspect ratio and flow direction cases can be attributed to the use of the plate dimensions $L$ or $W$ as the characteristiclength in the dimensionless quantities Nusselt number, $S^{*}$, and Reynolds number. From Fig. 3 it is seen that, for large Reynolds number, the Nusselt number becomes independent of the aspect ratio and flow direction and approaches a single asymptote, corresponding to the two-dimensional boundary-layer solution described earlier. However, for small values of Reynolds number, there is an order of magnitude difference between the results for $L / W=10$ when the flow is in the $L$ and $W$ direction. These differences in the diffusive limit change the shape of the Nusselt number vs Reynolds number 


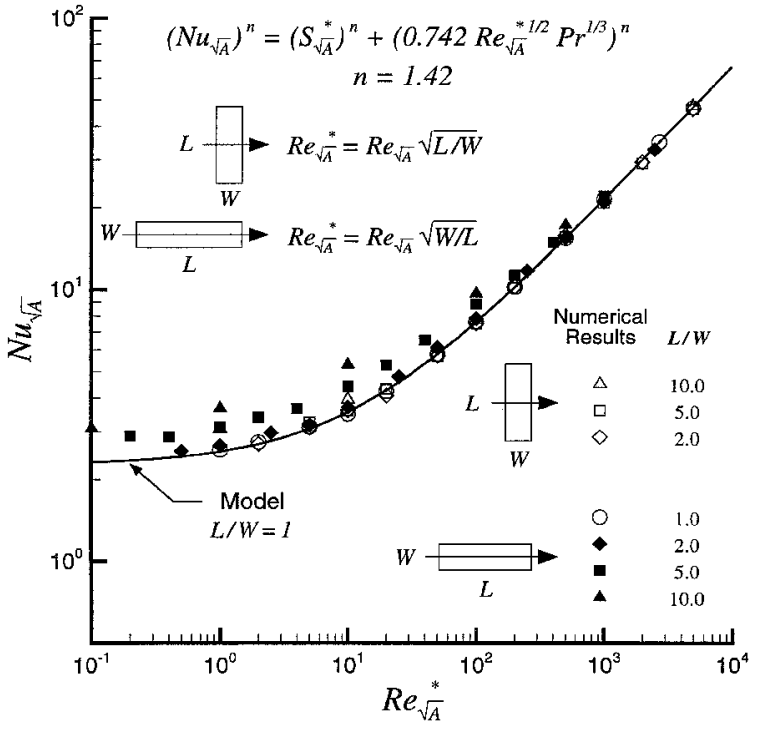

Fig. 4 Comparison of data and model with $\sqrt{ } A$ as characteristic length.

curves, requiring differentinterpolation parameters for the model to fit the data properly.

Previous research ${ }^{5}$ has shown that the use of the square root of the active surface area of the body as the characteristic length can reduce the variation of the results as a function of aspect ratio. In addition, the diffusive limit, Eqs. (8) and (9), is independent of the flow direction when $\sqrt{ } A$ is used as the characteristic length.

The data are recast using $\sqrt{ } A$ as the characteristic length, and the results, expressed as $N u_{\sqrt{ } A}$ are plotted as a function of $R e_{{ }^{*} A}^{*}$ in Fig. 4. The modified Reynolds number $R e^{*}{ }_{\sqrt{A}}$ has been introduced to account for flow direction effects in the boundary-layersolution. For bulk velocity parallel to the short dimension of the plate $W$, this modified Reynolds number is defined as

$$
R e_{\sqrt{A}}^{*}=R e_{\sqrt{A}} \sqrt{L / W}
$$

and for flow along the long plate dimension $L$,

$$
\operatorname{Re}_{\sqrt{A}}^{*}=\operatorname{Re}_{\sqrt{A}} \sqrt{W / L}
$$

The proposed model is recast in a similar manner,

$$
N u_{\sqrt{A}}=\left[\left(S_{\sqrt{A}}^{*}\right)^{n}+\left(0.742 \operatorname{Re} e_{\sqrt{A}}^{* \frac{1}{2}} \operatorname{Pr}^{\frac{1}{3}}\right)^{n}\right]^{1 / n}
$$

Equation (28) is plotted in Fig. 4 for the square plate only, $L=W$. Because the results for the square plate are unaffected by recasting the model in terms of $\sqrt{ } A$, the interpolation parameter used earlier, $n=1.42$, is retained.

From Fig. 4 it is seen that the variation between the data for different aspect ratios and flow directions has been reduced significantly. The data for the majority of test cases, including all aspect ratio cases for flow in the $W$ direction and $L / W \leq 2$ with flow in the $L$ direction, are in excellent agreement with the proposed model when $n=1.42$. The only substantial differences between the model and the data occur in the transition region for the large aspect ratio rectangles, $L / W=5.0$ and 10.0, with flow parallel to the long plate dimension $L$.

The proposed model using a fixed value of the interpolation parameter $n=1.42$ is compared with the CFD data for three aspect ratios, $L / W=2.0,5.0$, and 10.0, for flow in both directionsin Fig. 5 . In Fig. 5a the numerical results for $L / W=2.0$ with flow parallel to long and short plate dimensions are compared with the model. Through the use of the square root of area as the characteristiclength and the fixed interpolation parameter value, the model is independent of flow direction and can be represented on the plot by a single curve. The data in Fig. 5a also approach common asymptotes for

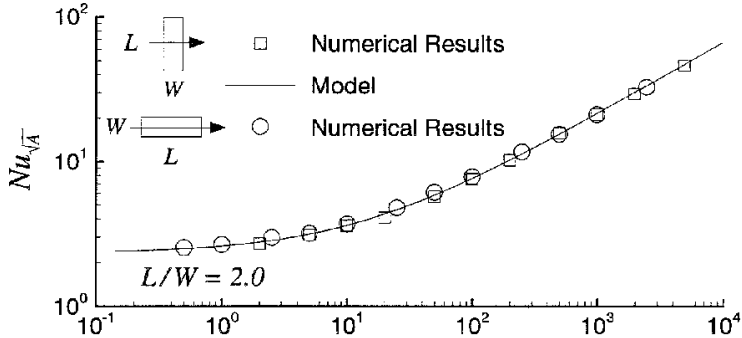

a) $L / W=2.0$

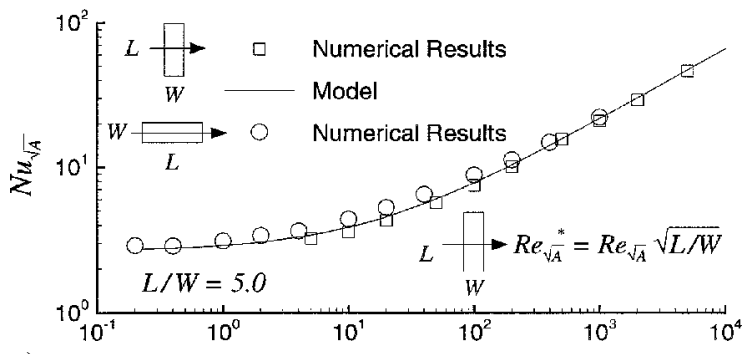

b) $L / W=\mathbf{5 . 0}$

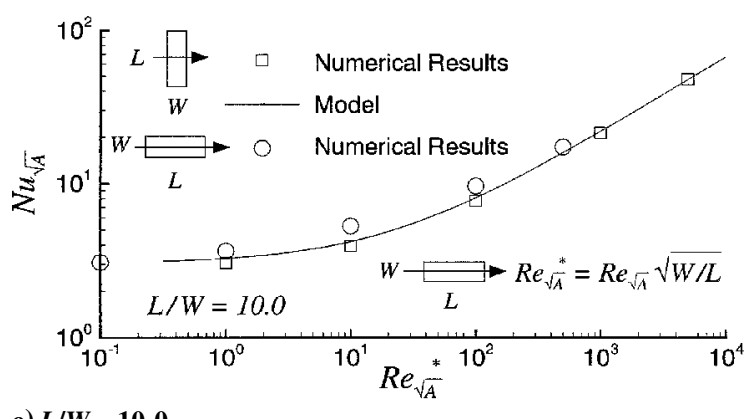

c) $L / W=10.0$

Fig. 5 Comparison of data and model.

large and small $R e^{*}{ }_{\sqrt{A}}$, but show small deviations of approximately $2-3 \%$ between the results for the two flow directions in the intermediate region, $R e_{\sqrt{ } A}^{*} \approx 10$.

The variations between the numerical data for the two flow directions is more evident for the $L / W=5.0$ case, with a maximum difference of $20 \%$ at $R e^{*}{ }^{*}=10$ as shown in Fig. 5b. These differences in the data for flow parallel to the long plate dimension $L$ and flow along the short dimension $W$ are the result of edge effects not accounted for in the model. For the finite plate, the boundary layer tends to become thinner near the edges due to diffusion, leading to enhanced heat transfer in these regions. This effect is most evident in the intermediate region, $5 \leq R e^{*}{ }_{{ }_{A}} \leq 50$, where boundary growth occurs quickly and the heat transfer is not conduction dominated. The edge effects tend to enhance heat transfer in cases where the bulk fluid velocity is parallel to the long plate dimension $L$, whereas the effects are minimized in cases with flow along the short plate dimension $W$, resulting in a lower $N u_{\sqrt{ } A}$. With a fixed value for the interpolation parameter, $n=1.42$, the model passes between the data, $7 \%$ above the lower data set and $13 \%$ below the upper data set at $R e^{\star}{ }_{\sqrt{A}}=10$

In Fig. 5c, the model with $n=1.42$ for $L / W=10$ once again passes through the middle of the numerical data for flow in the $L$ and $W$ directions. The variation between the data in the intermediate region for the two flow directions is the largest of the three cases, due to the increase in edge effects for the larger aspect ratio. The model underpredicts the data for flow parallel to the long plate dimension $L$ by a maximum of $26 \%$ at $R e^{*}{ }_{\sqrt{A}}=10$ and overpredicts the data for flow in the $W$ direction by a maximum of $6 \%$ at $R e^{*}{ }_{\sqrt{A}}=10$.

In general, the proposed model is in close agreement, within 6-7\%, of all of the data for flow in the $W$ direction and $L / W \leq 2$ for flow in the $L$ direction. The differences between the data and the model for $L / W=5.0$ and 10.0 are larger in the transition region due to edge effects. 
Temperature distribution data from the CFD simulations in the region surrounding the plate are shown in Figs. 6-8. Figures 6-8 contain two sets of temperature contours: isotherms in an $x y$ plane along the midplane of the plate, $z=W / 2$ or $z=L / 2$ (Figs. 6a, 7a, and $8 \mathrm{a}$ ) and isotherms in the $x z$ plane at the plate surface, $y=0$ (Figs. 6b, 7b, and 8b). Four differentflow rates are plotted in each set, starting with the diffusive limit, $R e \rightarrow 0$, followed by $R e=1,10$, and 100, where Reynolds number is defined by Eqs. (20) and (22).

Each of the sets of contour plots presented in Figs. 6-8 demonstrate the smooth transition between conduction-dominated (zero flow) and boundary-layerflow. At the diffusive limit, the isotherms extend radially out ward from the plate, quickly becoming sphericalshaped contours consistent with pure conduction into a half-space. As flow is introduced, the freestream velocity begins to affect the temperature distribution. At $R e=1$, the isotherms near the plate still resemble those in the diffusion problem, but the outer isotherms are distorted by the freestream velocity. At $R e=10$, the advection effects become stronger, a distinct thermal boundary layer begins to form, and the problem begins to display two-dimensional characteristics. Finally, at $R e=100$, two-dimensional boundary-layer

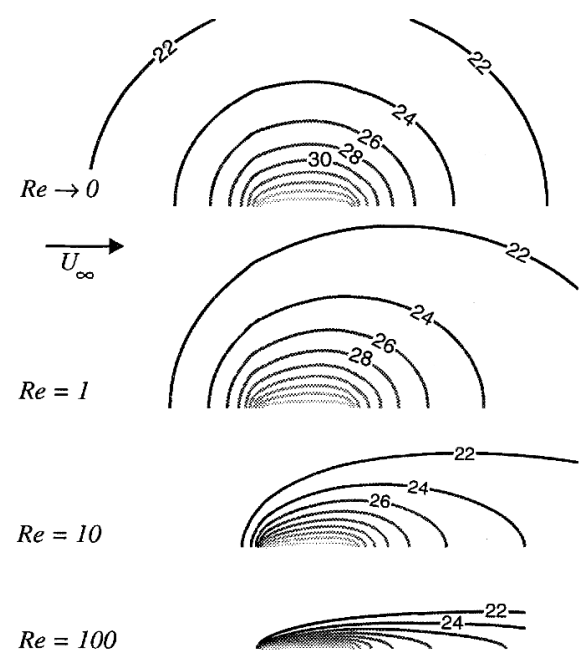

20.022 .024 .026 .028 .030 .032 .034 .036 .038 .0 a) Midplane, $z=L / 2$

$T\left({ }^{\circ} \mathrm{C}\right)$ behavior has been established, and diffusion in the $z$ direction perpendicular to the flow is minimized.

From the temperature contours for $L / W=5.0$ shown in Figs. 6 and 8 , it appears that transition from diffusion to boundary-layer flow occurs more slowly for flow in the $L$ direction than for flow in the $W$ direction. If the isotherms for $R e=100$ are compared for each flow direction it is seen that the penetration of the temperature field into the fluid is much larger in Fig. 8 for flow in the $L$ direction than in Fig. 6.

This behavior can be attributed to the use of the plate side dimensions as the characteristic length in Reynolds number. For each $L / W=5.0$ case, the value $R e=100$ can be recast in terms of the modified Reynolds number, $R e^{\star}{ }_{\text {A }}$ defined earlier. For flow parallel to the short plate dimension $W$, as shown in Fig. 6,

$$
R e=100, \quad R e_{\sqrt{A}}^{*}=500
$$

and for flow in the $L$ direction, as shown in Fig. 8,

$$
\operatorname{Re}=100, \quad \operatorname{Re}_{\sqrt{A}}^{*}=20
$$

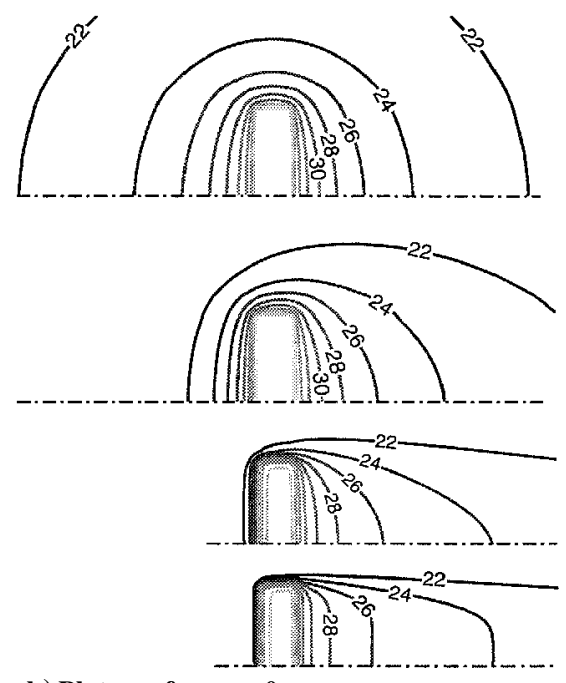

b) Plate surface, $y=0$

Fig. 6 Temperature distribution for $L / W=5.0$ with flow in $W$ direction.

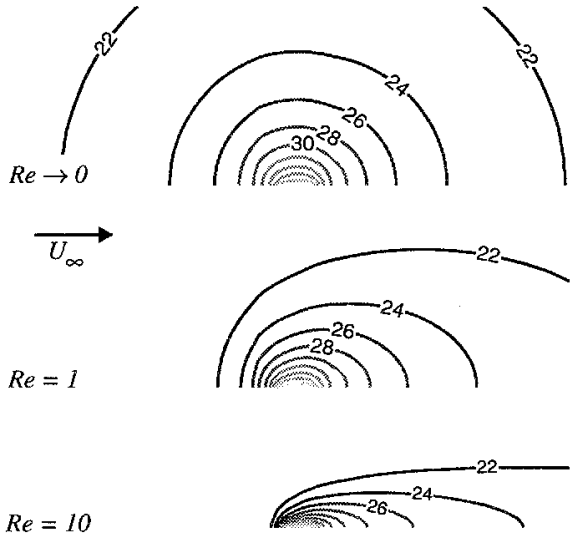

$R e=100$

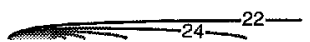

D.

20.022 .024 .026 .028 .030 .032 .034 .036 .038 .0 a) Midplane, $z=W / 2$ 

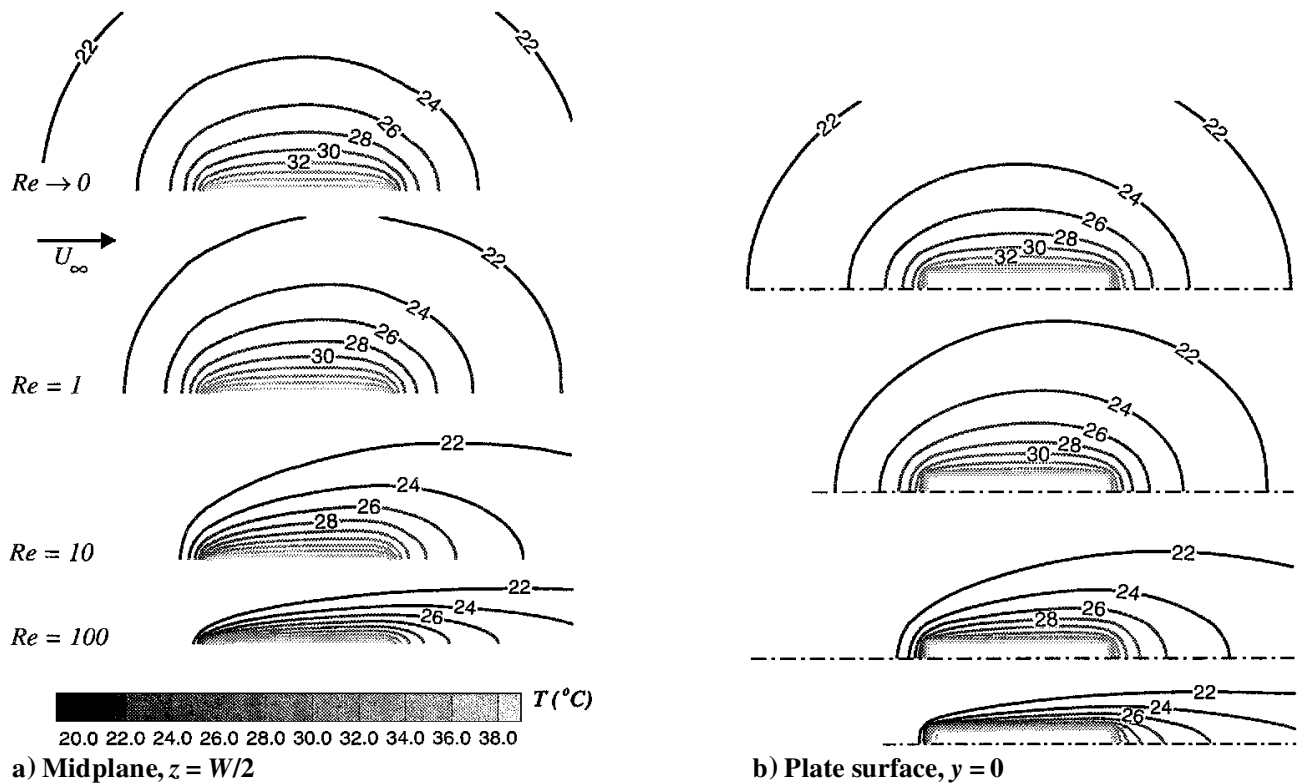

Fig. 8 Temperature distribution for $L / W=5.0$ with flow in $L$ direction.

Based on these values for the modified Reynolds number it can be concluded that at $R e=100$ the $L / W=5.0$ case shown in Fig. 8 is in the middle of the transition region, whereas the same case in Fig. 6 behaves according to the two-dimensional, boundary-layersolution.

\section{Summary and Conclusions}

A composite correlation equation has been developed that accurately predicts the average Nusselt number for laminar forced convection heat transfer from isothermal finite rectangular plates. The proposed model is valid for fluids with $\mathrm{Pr}>0.5$ and for a range of the Reynolds number $0<\operatorname{Re}_{\mathcal{L}}<5000$ for flow in both the $W$ and $L$ directions.

It has been demonstrated that the use of the square root of the active surface area as the scale length in the dimensionless parameters reduces the effects of aspect ratio and flow direction on the solution. Using $\sqrt{ } A$ as the characteristic length in the correlation equation reduces the range of the interpolation parameter to a single value for all aspect ratios and flow directions.

Accurate numerical results have been presented for the Nusselt number for the aspect ratios $L / W=1.0,2.0,5.0$, and 10.0 in the range $0<R e<5000$ with flow in both the $W$ and $L$ directions. When the plate side length, $L$ or $W$, is used as the scale length, and the interpolation parameter is determined using the correlations provided, the proposed model and the data have a maximum percent difference of $4.5 \%$ and an rms percent difference of $1.9 \%$.

Contour plots for the temperature distribution in the region surrounding the plate have been generated using the data from CFD simulations and are presented for three cases. The isotherms in these contour plots demonstrate the smooth transition between the diffusive limit, $R e \rightarrow 0$, and the two-dimensional, laminar boundarylayer solution.

\section{Acknowledgments}

The first author acknowledgesthe financial support of the Natural Sciences and Engineering Research Council of Canada under Grant A7455. The authorsalso thank Materials and ManufacturingOntario for their continued support.

\section{References}

${ }^{1}$ Schlichting, H., Boundary Layer Theory, 6th ed., McGraw-Hill, New York, 1968, pp. 278-286.

${ }^{2}$ Bejan, A., Convective Heat Transfer, Wiley, New York, 1984, pp. 46-52.

${ }^{3}$ Kays, W. M., and Crawford, M. E., Convection Heat and Mass Transfer, 2nd ed., McGraw-Hill, New York, 1980, pp. 134-139.

${ }^{4}$ Refai Ahmed, G., and Yovanovich, M. M., "Analytical Method for Forced Convection from Flat Plates, Circular Cylinders, and Spheres," Journal of Thermophysics and Heat Transfer, Vol. 9, No. 3, 1995, pp. 516523.

${ }^{5}$ Yovanovich, M. M., "Dimensionless Shape Factors and Diffusion Lengths of Three-Dimensional Bodies," Proceedings of the ASME/JSME Thermal Engineering Joint Conference, Vol. 1, American Society of Mechanical Engineers, New York, 1995, pp. 103-113.

${ }^{6}$ Schneider, G. E., "Thermal Resistance Due to Arbitrary Dirichlet Contacts on a Half-Space," Thermophysics and Thermal Control, Vol. 65, Progress in Astronautics and Aeronautics, AIAA, New York, 1978, pp. 103119 .

${ }^{7}$ Yovanovich, M. M., Lee, S., and Gayowsky, T. J., "Approximate Analytical Solution of Laminar Forced Convection from an Isothermal Plate," AIAA Paper 92-0248, Jan. 1992.

${ }^{8}$ Churchill, S. W., and Usagi, R., "A General Expression for the Correlation of Rates of Transfer and Other Phenomena," AIChE Journal, Vol. 18 No. 6, 1972, pp. 1121-1128.

${ }^{9}$ FLOTHERM, V. 1.4, Flomerics Inc., Marlborough, MA, 1998.

${ }^{10}$ Patankar, S. V., Numerical Heat Transfer and Fluid Flow, McGrawHill, New York, 1980, pp. 126-135. 